\title{
Entrapment of a vaginal ring pessary: Case report and review of the literature
}

\author{
Z Abdool, MMed (O\&G), FCOG (SA) \\ Department of Obstetrics and Gynaecology, Steve Biko Academic Hospital and Faculty of Health Sciences, University of Pretoria, South Africa
}

Corresponding author: Z Abdool (zeelha.abdool@up.ac.za)

The use of vaginal pessaries for symptomatic pelvic organ prolapse (POP) is well established. Recently pessaries have been offered routinely as a first-line treatment option for symptomatic POP, and in patients with medical comorbidity, those who are unfit for surgical intervention, and young women who still wish to bear children. The favourable physical and chemical properties of silicone have made pessaries safer to use for the treatment of POP. Complications associated with neglected pessaries are well documented, and it is probable that complications are rare when pessary care is regular. We present a case of partial encapsulation of a ring pessary despite regular follow-up at a tertiary urogynaecological unit, and review the literature pertaining to early entrapment of vaginal ring pessaries.

S Afr J Obstet Gynaecol 2015;21(1):14-15. DOI:10.7196/SAJOG.905

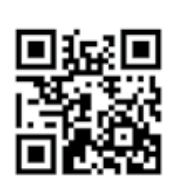

A variety of pessaries are available, but it is evident from the literature that the ring pessary is the most common type prescribed, regardless of compartmental defect. Although the early literature commonly reserved their use for patients who declined surgery, were unfit for surgery or required interim relief while awaiting surgery, and for young women who wanted to fall pregnant, their use as first-line treatment for symptomatic pelvic organ prolapse (POP) is currently common clinical practice among gynaecologists and allied health clinicians (nurses and physiotherapists). ${ }^{[1-4]}$ Using a variety of questionnaires, several studies have demonstrated both a statistically and clinically significant improvement in prolapse, urinary and bowel sympotoms, sexual activity, and general quality of life. ${ }^{[5-8]}$ Handa and Jones ${ }^{[9]}$ reported a significant improvement in stage of prolapse $(p=0.045)$ in a small group of 19 women who were fitted with a ring pessary for at least 1 year, and Matsubara and Ohki ${ }^{[10]}$ reported correction/reversal of uterine prolapse in six women 42 months after removal of the ring pessary, suggesting a therapeutic effect.

Major complications associated with neglected ring pessaries, such as fistulas (vesicovaginal, rectovaginal), vaginal vault perforation, pessary incarceration, urosepsis and pessary entrapment/ embedment, are well noted in the literature. ${ }^{[1]}$ Lone et al. ${ }^{[3]}$ reported an overall minor complication rate of $12.1 \%$ (pain/discomfort, excoriation/bleeding, disimpaction/constipation) in symptomatic patients followed up over a 5-year period. Entrapment, also frequently referred to as embedment, is a common complication reported mainly with neglected and forgotten ring pessaries. We present a case of a ring pessary entrapped in a band of vaginal tissue despite frequent pessary care at a tertiary urogynaecology clinic.

\section{Case report}

A 64-year-old woman, para 7, gravida 7, presented to the urogynaecology clinic for her routine 3 - 6-monthly pessary review visit. She had no current complaints and was satisfied using the ring pessary as a treatment option for symptomatic POP. On clinical examination the clinician failed to remove the ring pessary, which was retained.
The patient had initially been referred to the urogynaecology clinic for symptomatic POP in August 2009. She had had seven previous normal vaginal deliveries with no complications. Examination revealed anterior compartment prolapse, cystocele POP-Q stage 2. The patient was offered either surgical intervention or a pessary as a treatment option. She opted for the latter. A $70 \mathrm{~mm}$ (size 4) siliconebased ring pessary (without support) was inserted. We routinely review the patient 2 weeks after insertion and then at 3-monthly intervals. She continued to present for her routine review on a 3 6-monthly basis as she could not attend at 3-monthly intervals. She noted that the pessary had significantly improved her prolapse symptoms. At each visit the pessary was removed and cleaned and the vaginal mucosa inspected with a Cusco speculum, as per protocol. Vaginal oestrogen cream was prescribed intermittently as deemed necessary by the attending clinician.

On further examination of the retained pessary, a thick band of granulation tissue (approximately $2 \mathrm{~cm}$ ) had grown over the pessary

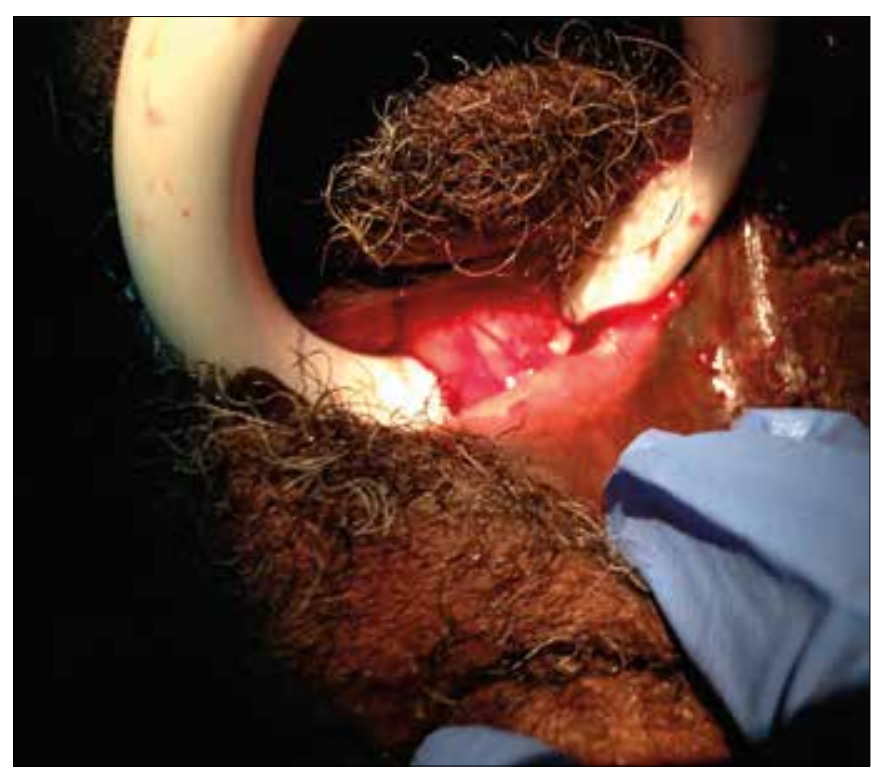

Fig. 1. Thick band of granulation tissue causing entrapment of the pessary. 
(Fig. 1). Local anaesthetic was injected into the granulation tissue and it was incised in the examination room with no complications and no need for further analgesia. The vagina was packed and the patient was monitored for vaginal bleeding for 4 hours after the procedure. She was discharged home without pessary reinsertion, with instructions to use vaginal oestrogen cream daily. She was reviewed 6 weeks later, and requested pessary reinsertion after declining surgical intervention. She gave written consent for publication of this case report.

\section{Discussion}

Vaginal pessaries form an important part of the treatment armamentarium for general practitioners, gynaecologists and nurses. They are prescribed as first-line treatment for prolapse by $77 \%$ of American urogynaecologists, $86.7 \%$ of UK gynaecologists and $24 \%$ of South African gynaecologists. ${ }^{[2,12]}$ The simple ergonomic design of the ring pessary is appealing to both the healthcare provider and the patient, which may explain its popularity. It can be removed and replaced easily by both clinician and patient, and its use is not dependent on stage and type of prolapse, although some manufacturers recommend the ring pessary for first- and second-degree prolapse.

Major complications with ring pessaries rarely occur with frequent follow-up, and minor clinical issues such as vaginal discharge, odour, irritation and erosions are often tolerated by informed patients. ${ }^{[11]}$ Lone et al. ${ }^{[3]}$ performed a large prospective study to identify complications of pessary use and reasons for discontinuation over a period of 5 years. It was noted that most complications become apparent within the first 6 months after pessary insertion, and minor complications such as vaginal discharge, odour and excoriation/bleeding can be managed conservatively with local oestrogen application, a pessary 'holiday' and a change in pessary size at reinsertion. These patients were reviewed 6-monthly at a urogynaecology clinic and no major complications were noted in patients who continued use at 5 years $(n=130)$.

Vaginal ring entrapment, also referred to as encapsulation, embedment or incarceration, is a recognised complication that is commonly reported in older patients who have either forgotten or neglected their pessary care routine. Early entrapment of a ring pessary in patients who attend for 3 - 6-monthly followup is uncommon. The literature reports three cases $^{[13-15]}$ of early entrapment of vaginal ring pessaries at 4 and 5 months post review/ insertion. The case reported by Thornton and Harrison ${ }^{[13]}$ was a 59-year-old woman with procidentia awaiting surgical intervention. A $95 \mathrm{~mm}$ polyvinyl (PVC) ring pessary was inserted, and it was removed under general anaesthesia 4 months later because of incarceration. Whitworth et al. ${ }^{[14]}$ reported a 73-year-old patient with three-compartment prolapse who had been using an $80 \mathrm{~mm}$ PVC ring pessary for 10 years (poor surgical candidate). Five months after the last visit the pessary was described as incarcerated and vaginal tissue was excised uneventfully. In 2007 Govind and Lahki ${ }^{[15]}$ reported entrapment of an $80 \mathrm{~mm}$ polyethylene (Portex) ring pessary only 4 months after insertion in a 75 -year-old patient with a cystourethrocele. Although these cases were easily treated, in severe cases with neglected or forgotten pessaries a bone cutter can be used in theatre to remove the entrapped ring. ${ }^{[15]}$
Reasons postulated for early vaginal ring pessary entrapment may be related to the type of pessary material (vulcanised rubber v. silicone-based pessaries), concomitant vaginal atrophy, and inherent local tissue reaction in response to chronic vaginal irritation. Watch-spring and vulcanite-based (combination of rubber and sulphur) pessaries were predominantly used in the early 1900s, and polythene-based as opposed to silicone-based pessaries in the mid1900s. These were not easily compressible and produced marked tissue reactions. Polythene, also referred to as 'common plastic', is composed of a long chain of hydrocarbons with differing molecular weights, as opposed to silicone, which consists of a silicone and oxygen backbone to which organic groups such as methyl, ethyl or phenyl are attached. The chain length, cross-linking and side-group attachment determine the properties and composition, e.g. gas, liquid, gel, rubber. The favourable physical and chemical properties of silicone, i.e. bioinert, stable and readily flexible, make it the best material currently available for pessary manufacture.

\section{Conclusion}

At present there are no universally standardised pessary protocols, and clinicians depend on expert opinion and manufacturer recommendations. However, it is widely agreed that a pessary clinic routinely review patients at $3-6$-monthly intervals. At these followup visits, the vagina is carefully inspected for erosions/abrasions, especially the lateral vaginal fornices and apical area. The pessary should be cleaned with soap and water. If patients are comfortable about handling the pessary and performing self-care, intervals may be extended to 6 - 12-monthly intervals. All patients should be educated about self-care and the significance of strictly timed follow-up visits.

1. Bugge C, Hagen S, Thakar R. Vaginal pessaries for pelvic organ prolapse and urinary incontinence A multiprofessional survey of practice. Int Urogynecol J Pelvic Floor Dysfunct 2013;24(6):10171024. [http://dx.doi.org/10.1007/s00192-012-1985-7]

2. Cundiff GW, Weidner AC, Visco AG, Bump RC, Addison WA. A survey of pessary use by members of the American Urogynecologic Society. Obstet Gynecol 2000;95(6):931-935. [http://dx.doi. org/10.1016/S0029-7844(00)00788-2]

3. Lone F, Thakar R, Sultan AH, Karamalis G. A 5-year prospective study of vaginal pessary use for pelvic organ prolapse. Int J Gynaecol Obstet 2011;114(1):56-59. [http://dx.doi.org/10.1016/j. ijgo.2011.02.006]

4. Weber AM, Richter HE. Pelvic organ prolapse. Obstet Gynecol 2005;106(3):615-634. [http://dx.doi. org/10.1097/01.AOG.0000175832.13266.bb]

5. Cundiff GW, Amundsen CL, Bent AE, et al. The PESSRI study: Symptom relief outcomes of a randomized crossover trial of the ring and Gellhorn pessaries. Am J Obstet Gynecol 2007;196(4):405.e1-405.e8. [http://dx.doi.org/10.1016/j.ajog.2007.02.018]

6. Komesu YM, Rogers RG, Rode MA, et al. Pelvic floor symptom changes in pessary users. Am J Obstet Gynecol 2007;197(6):620.e1-620.e6. [http:dx.doi.org/10.1016/j.ajog.2007.08.013]

7. Kuhn A, Bapst D, Stadlmayr W, Vits C, Mueller M. Sexual and organ function in patients with symptomatic prolapse: Are pessaries helpful? Fertil Steril 2009;91(5):1914-1918. [http://dx.doi. org/10.1016/j.fertnstert.2008.02.142]

8. Abdool Z, Thakar R, Sultan AH, Oliver R. Prospective evaluation of outcome of vaginal pessaries versus surgery in women with symptomatic pelvic organ prolapse. Int Urogynecol 2011;22(3):273-278. [http://dx.doi.org/10.1007/s00192-010-1340-9]

9. Handa VL, Jones M. Do pessaries prevent the progression of pelvic organ prolapse? Int Urogynecol J Pelvic Floor Dysfunct 2002;13(6):349-351, discussion 352. [http://dx.doi.org/10.1007/ s0019202000078]

10. Matsubara S, Ohki Y. Can a ring pessary have a lasting effect to reverse uterine prolapse even after its removal? J Obstet Gynaecol Res 2010;36(2):459-461. [http://dx.doi.org/10.1111/j.14470756.2009.01162.x]

11. Arias BE, Ridgeway B, Barber MD. Complications of neglected vaginal pessaries: Case presentation and literature review. Int Urogynecol J Pelvic Floor Dysfunct 2008;19(8):1173-1178. [http://dx.doi. org/10.1007/s00192-008-0574-2]

12. Abdool Z. Evaluation of vaginal pessary use by South African gynaecologists. S Afr J Obstet Gynaecol 2011;17(3):64-67.

13. Thornton CA, Harrison RF. Unusually rapid incarceration of a polyvinyl ring pessary. Ir J Med Sc 1977;146(4):116. [http://dx.doi.org/10.1007/BF03030943]

14. Whitworth JS, Thijs I, Bhal PS. Rapid incarceration of a ring pessary with its safe and immediate removal. J Obstet Gynaecol 2002;22(2):225-226.

15. Govind A, Lakhi N. The enigma of early entrapment of vaginal ring pessaries. J Obstet Gynaecol 2007;27(4):451-452. [http://dx.doi.org/10.1080/01443610701383258] 